\title{
Sensorless Speed Control of Traveling Wave Ultrasonic Motor
}

\author{
Markus Flueckiger, Student Member IEEE, Matteo Bullo, Yves Perriard, Senior Member IEEE \\ Integrated Actuators Laboratory - LAI \\ Ecole Polytechnique Fédérale de Lausanne - EPFL \\ CH - 1015 LAUSANNE - SWITZERLAND \\ Fax : +41216932050 \\ Email : markus.flueckiger@epfl.ch
}

\begin{abstract}
Ultrasonic motors are a good alternative to electromagnetic motors in medical robotics, since they are electromagnetically compatible. Estimating speed instead of using encoders reduces cost and dimension of the robot on the one hand and increases reliability on the other hand. However, no sensorless speed controller is yet industrialized. Analytical models of the traveling wave ultrasonic motor being too complex to be exploited for sensorless control purpose, we suggest speed estimation based on artificial neural networks. The artificial neural network is designed based on a sensitivity analysis using design of experiments methods. Factorial designs have been chosen to find out the effects of each input factor, but also the effect of their interactions. First results show that speed estimation using artificial neural networks is a promising approach. The artificial neural network optimized with design of experiments methods is a valid model of the traveling wave ultrasonic motor to estimate speed.
\end{abstract}

\section{Motivation}

We are developing haptic interfaces compatible with functional magnetic resonance imaging (fMRI) for neuroscience studies. A one degree of freedom prototype actuated by a traveling wave ultrasonic motor (USM) using load and speed sensors for operation under admittance control has been developed. This device can be used in conjunction with fMRI, providing torque and motion feedback simultaneously with imaging without deteriorating imaging quality [1]. While the load torque must be measured for experimental purposes, a sensorless speed controller would reduce complexity and favor miniaturization of the device and therefore facilitate the intended construction of multiple degree of freedom systems.

\section{Traveling Wave Ultrasonic Motor}

\section{A. Characteristics}

USM are well suited to haptic interfaces in medical applications [2], because on one hand, they present a high power density and a high output torque at low speed, and on the other hand due to their magnetic compatibility [3].

Figure 1 shows a schematic of the used USM. There are two stages of energy conversion :

- In the stator, electrical energy is converted to strain energy through piezoceramics, where the supply signal induces mechanical vibrations.

- The frictional impact at the stator-rotor interface transforms these ultrasonic vibrations into rotary movement of the stator.

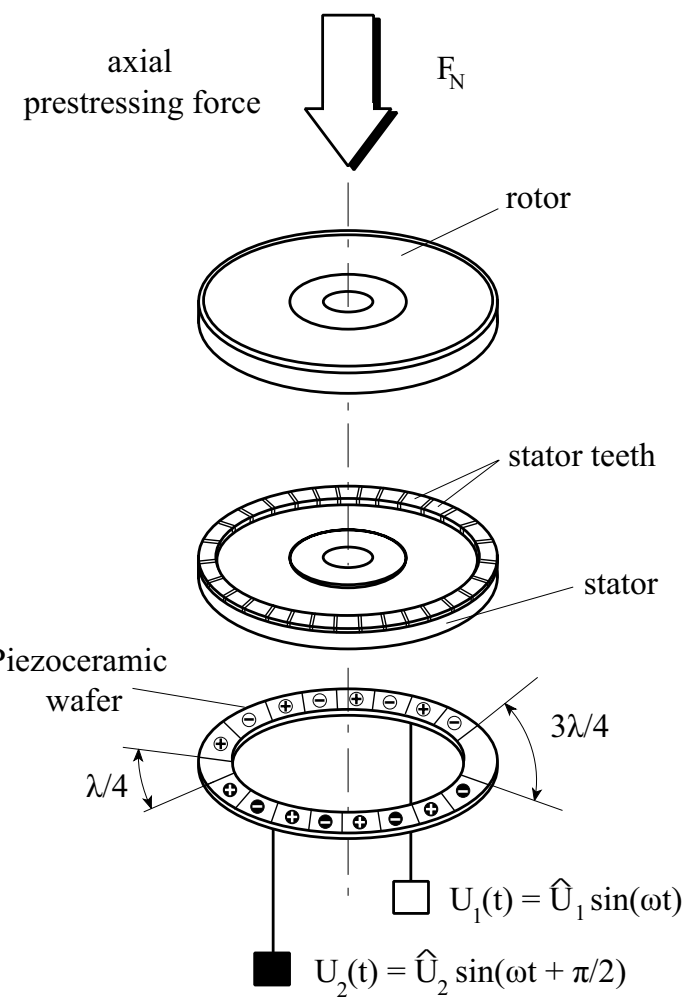

Fig. 1. Schematic of the motor structure. The piezoceramique wafer is etched into sectors according to wavelength $(\lambda)$ of traveling wave. $\oplus$ or $\ominus$ indicate poling direction of sector.

The detailed operating principle of the USM is explained in [4] and [5].

\section{B. Modeling}

While the linear characteristics of free vibration in the stator in the first stage can be modeled by equations of motion, the phenomenon at the stator-rotor interface inherently displays non-linear dynamics and modeling becomes complex.

Important contributions are the equivalent circuit model [4], finite element approaches [6] or the analytical model incorporating the stator-rotor interface forces and predicting the resulting motor performance as a function of design parameters [7]. 
Due to their complexity, these models are either difficult or even impossible to exploit for sensorless control purposes. Hence, we decided to model the actuator using artificial neural networks (ANN).

In an earlier work [8] we studied the performance of the USM. Namely, we investigated efficiency and optimized the axial prestressing force between stator and rotor (cf. Figure $1)$, which is chosen according to the results obtained in [8]. Here, we consider the motor to be a black box. We use Design of Experiment (DoE) techniques to identify how the input parameters influence the output speed and in which manner the inputs interact. We consider only the linear regions of the experimental domain where the motor's behavior is useful. In such a way, a linear model can represent the motor characteristics well enough. The results obtained in this process show us which input parameters are useful as inputs to the ANN. The ANN is then trained using the DoE data obtained with a test bench as well as experimental data from the prototype of the haptic interface presented in [1].

\section{SEnsitivity AnAlysis}

We know that the output of the ANN will be the motor speed. But the possible input parameters must be the subject of an evaluation process to understand which of them are suitable to achieve the most accurate speed prediction at minimal cost. Sensitivity analysis can be used to determine factors that have a large influence on the output and also to identify interactions between factors. These findings may show that it is sufficient to use as inputs some subset of the correlated variables.

\section{A. Design of Experiments}

The sensitivity analysis using DoE distinguishes the present project from earlier research done on the subject [9]. It allows one to find the ideal input parameters to the neural network while optimizing the accuracy of the output speed prediction. There exists a large variety of experimental designs [10]. The sensitivity analysis using the DoE methods is divided into the following tasks :

- Choice of possible input parameters, and definition of their range.

- Data acquisition, by varying the input parameters according to the defined range.

- Determination of the influence on the output and correlation of the input parameters.

The last and crucial step is carried out by applying the DoE formalism implemented in Matlab to the experimental data. All measurable input parameters are tested for their influences on the output and their correlation. If the influence on the output of a parameter is below a specific value or if its correlation with another, more critical parameter, is above a defined value, it will not be used as an input for the ANN.

\section{B. Factorial Design and Polynominal Linear Regression}

This method allows us to study the effect of each factor on the response variable, while requiring fewer observations than by conducting separate experiments for each factor independently. This is achieved by choosing the points of measure at the edge of the multi-dimensional domain defined by the input parameter ranges and fitting the acquired data to an appropriate polynomial function corresponding to a Taylor series of the model being analyzed (Equation 1). It also allows studying the effect of the interaction between factors on the response variable. Full factorial design allows for determining all coefficients of a linear model with all possible interactions within $2 \mathrm{~N}$ runs.

The coefficients are found by polynomial linear regression (least square fitting) from the data obtained according to the designed experiment.

$$
\begin{aligned}
Y(x)= & a_{0}+\sum_{i=1}^{N} a_{i} x_{i}+\sum_{i \neq j}^{N} a_{i j} x_{i} x_{j}+\cdots \\
& \sum_{i \neq j \neq k}^{N} a_{i j k} x_{i} x_{j} x_{k}+a_{i \ldots N} x_{i} \ldots x_{N}
\end{aligned}
$$

The coefficients $a_{0}, a_{1} \ldots$ are called the half effects of the $x_{i}$ factors, which are classified as follows :

$a_{0}$ constant effect (equal to the experiments mean)

$a_{i}$ main half effects

$a_{i j}$ first order interaction half effects

$a_{i j k}$ second order interaction half effects

If the response of the system is organized in a vector $R=$ $\left[R_{1} R_{2} \ldots R_{n}\right]^{T}$ and the coefficients of the model in a vector $C=\left[a_{0} a_{1} a_{2} \ldots a_{12} \ldots a_{12 \ldots n}\right]^{T}$, then the system of equations is written down and solved by the following way :

$$
X C=R \Rightarrow C=\left(X^{T} X\right)^{-1} X^{T} R
$$

\section{RESULTS OF THE SENSITIVITY ANALYSIS}

\section{A. Data Analysis and ANOVA}

There are two main methods of data analysis :

- The comparison of the data with a statistical distribution

- The comparison of a subset of the data with another subset of the data

We are interested in comparing the effects with the residual error. Therefore, we opt for the second approach by applying an analysis of variance (ANOVA) on the modeling data. Let $n$ be the number of sets of identical observations within each of $K$ factors and $y_{i j}$ be the $j$ th observation within factor $i$. We balance the ANOVA by restricting $n$ to be the same for each factor.

The total sum of squares :

$$
S S T \equiv \sum_{i=1}^{k} \sum_{j=1}^{n}\left(y_{i j}-\overline{\bar{y}}\right)^{2}
$$

$$
=\sum_{i=1}^{k} \sum_{j=1}^{n} y_{i j}^{2}-\frac{\left(\sum_{i=1}^{k} \sum_{j=1}^{n} y_{i j}\right)^{2}}{K n}
$$


ANOVA global

\begin{tabular}{|r|c|c|c|c|c|}
\hline Source & SS & df & MS & F & $\mathrm{p}$ \\
\hline Model & 1297505.22 & 36 & 36041.81 & 101.38 & 0.00 \\
\hline Residual & 8888.03 & 25 & 355.52 & & \\
\hline Test & 1306393.25 & 61 & & & \\
\hline
\end{tabular}

ANOVA model factors

\begin{tabular}{|c|c|c|c|c|c|}
\hline Source & SS & df & MS & $\mathrm{F}$ & $\mathrm{p}$ \\
\hline Iph & 477413.32 & 1 & 477413.32 & 1342.85 & 0.00 \\
\hline Uph & 156202.14 & 1 & 156202.14 & 439.36 & 0.00 \\
\hline Itot & 389072.18 & 1 & 389072.18 & 1094.37 & 0.00 \\
\hline $\mathrm{M}$ & 184274.89 & 1 & 184274.89 & 518.32 & 0.00 \\
\hline $\mathrm{f}$ & 12960.12 & 1 & 12960.12 & 36.45 & 0.00 \\
\hline phi & 906.65 & 1 & 906.65 & 2.55 & 12.28 \\
\hline Uo & 661.86 & 1 & 661.86 & 1.86 & 18.46 \\
\hline Iph*Iph & 4519.94 & 1 & 4519.94 & 12.71 & 0.15 \\
\hline Iph*Uph & 5304.48 & 1 & 5304.48 & 14.92 & 0.07 \\
\hline Iph*Itot & 3740.86 & 1 & 3740.86 & 10.52 & 0.33 \\
\hline Iph*M & 26893.81 & 1 & 26893.81 & 75.65 & 0.00 \\
\hline Iph*f & 305.03 & 1 & 305.03 & 0.86 & 36.32 \\
\hline Iph*phi & 4038.34 & 1 & 4038.34 & 11.36 & 0.24 \\
\hline Iph*Uo & 1522.80 & 1 & 1522.80 & 4.28 & 4.90 \\
\hline Uph*Uph & 27.21 & 1 & 27.21 & 0.08 & 78.43 \\
\hline Uph*Itot & 2831.74 & 1 & 2831.74 & 7.97 & 0.92 \\
\hline Uph*M & 6793.38 & 1 & 6793.38 & 19.11 & 0.02 \\
\hline Uph*f & 1710.36 & 1 & 1710.36 & 4.81 & 3.78 \\
\hline Uph*phi & 625.64 & 1 & 625.64 & 1.76 & 19.66 \\
\hline Uph*Uo & 144.41 & 1 & 144.41 & 0.41 & 52.97 \\
\hline Itot*Itot & 3191.91 & 1 & 3191.91 & 8.98 & 0.61 \\
\hline Itot*M & 189.53 & 1 & 189.53 & 0.53 & 47.21 \\
\hline Itot*f & 1487.05 & 1 & 1487.05 & 4.18 & 5.15 \\
\hline Itot*phi & 6570.12 & 1 & 6570.12 & 18.48 & 0.02 \\
\hline Itot*Uo & 452.15 & 1 & 452.15 & 1.27 & 27.01 \\
\hline $\mathrm{M}^{*} \mathrm{M}$ & 1578.93 & 1 & 1578.93 & 4.44 & 4.53 \\
\hline $\mathrm{M} * \mathrm{f}$ & 148.35 & 1 & 148.35 & 0.42 & 52.42 \\
\hline M*phi & 220.63 & 1 & 220.63 & 0.62 & 43.82 \\
\hline M*Uo & 0.00 & 1 & 0.00 & 0.00 & 99.81 \\
\hline $\mathrm{f}^{* \mathrm{f}}$ & 449.77 & 1 & 449.77 & 1.27 & 27.14 \\
\hline f*phi & 28.89 & 1 & 28.89 & 0.08 & 77.80 \\
\hline f*Uo & 92.38 & 1 & 92.38 & 0.26 & 61.47 \\
\hline phi*phi & 511.47 & 1 & 511.47 & 1.44 & 24.16 \\
\hline phi*Uo & 16.11 & 1 & 16.11 & 0.05 & 83.31 \\
\hline Uo*Uo & 55.77 & 1 & 55.77 & 0.16 & 69.54 \\
\hline Residual & 8888.03 & 25 & 355.52 & & \\
\hline Test & 1306393.25 & 61 & & & \\
\hline
\end{tabular}

TABLE I

THE TABLE SHOWS SUM OF SQUARES (SS), DEGREES OF FREEDOM (DF), MEAN OF SQUARES (MS), THE F-RATIO (F) AND THE PROBABILITY GETTING THE SAME RATIO RANDOMLY (P). THUS THE ANOVA INDICATES WHICH EFFECTS ARE SIGNIFICANT.
The sum of squares of the model :

$$
S S M \equiv \frac{1}{n} \sum_{i=1}^{k}\left(\sum_{j=1}^{n} y_{i j}\right)^{2}-\frac{1}{K n}\left(\sum_{i=1}^{k} \sum_{j=1}^{n} y_{i j}\right)^{2}
$$

The sum of squares of the residual :

$$
S S R \equiv \sum_{i=1}^{k} \sum_{j=1}^{n}\left(y_{i j}-\bar{y}_{i}\right)^{2}=S S T-S S M
$$

where $\overline{y_{i}}$ is the mean of observations within a factor and $\overline{\bar{y}}$ is the mean of means [11].

The results of the ANOVA are shown in Table I. We calculate the F-Ratio (F) of the mean squared values (MS) of model and residual. It is expected that the mean square of a significant effect will be significantly greater than the mean square of the residual errors. The evaluation of the ratio is done in an objective way by comparing it with the probability of such a ratio between two random variables following a $\chi^{2}$ distribution. This comparison is then done using the Fcumulative distribution function, giving the probability of getting the same ratio randomly :

$$
\begin{aligned}
& p=1-F\left(x \mid \nu_{1}, \nu_{2}\right) \\
&=1-\int_{x}^{0} \frac{\Gamma\left[\frac{\left(\nu_{1}+\nu_{2}\right.}{2}\right]}{\Gamma\left(\frac{\nu_{1}}{2}\right) \Gamma\left(\frac{\nu_{2}}{2}\right)}\left(\frac{\nu_{1}}{\nu_{2}}\right)^{\frac{\nu_{1}}{2}} \frac{t^{\frac{\nu_{1}-2}{2}}}{\left[1+\left(\frac{\nu_{1}}{\nu_{2}}\right) t\right]^{\frac{\nu_{1}+\nu_{2}}{2}}} d t
\end{aligned}
$$

Here, the higher the probability of $p$, the less the effect is significant. The distribution depends on the degrees of freedom $\nu_{1}$ (the considered effect) and $\nu_{2}$ (the residual).

\section{B. Test Bench}

A schematic representation of the test bench is shown in Figure 2.

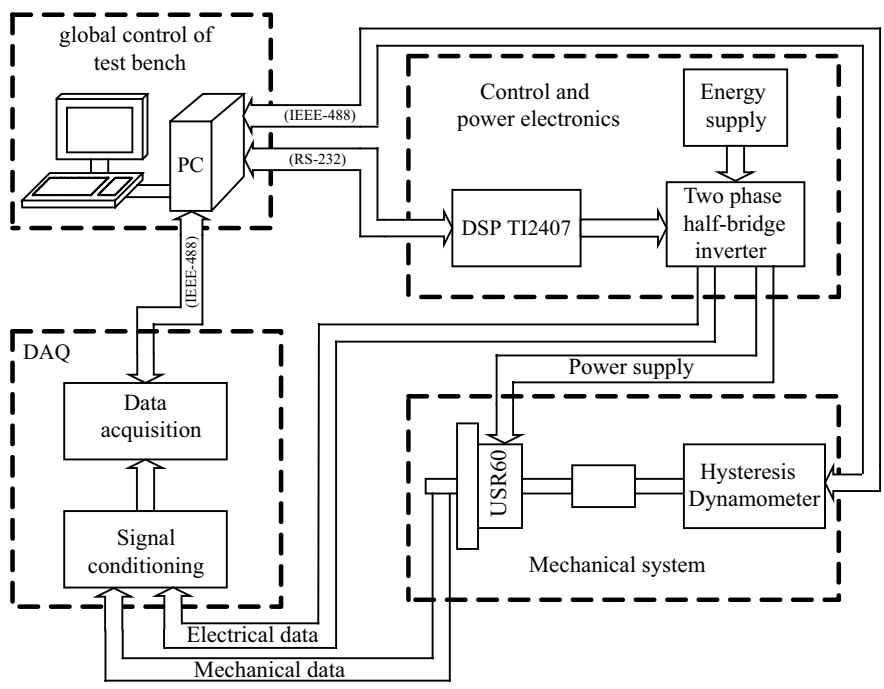

Fig. 2. Test bench.

The mechanical system is composed of a Shinsei USR 60 USM mounted on a aluminium support and coupled to a hysteresis dynamometer. The dynamometer delivers load torque independently of motor speed and therefore allows for measuring the motor characteristics on the full range from no load to motor blocking. Speed is measured with a rotary optical encoder.

A TI TMS2407 Microcontroller is used to compute the control signal; the power conversion circuit described in [12] generates the two alternating voltages which drive the motor. 


\section{Linear Model of USM}

To represent the system characterized by the input parameters in Table II with a linear model, we use a method including also quadratic effects. The space spanned by the factorial design was augmented with star points along the axes, and center points at the origin to form a central composite design. Second order and higher interaction effects were neglected.

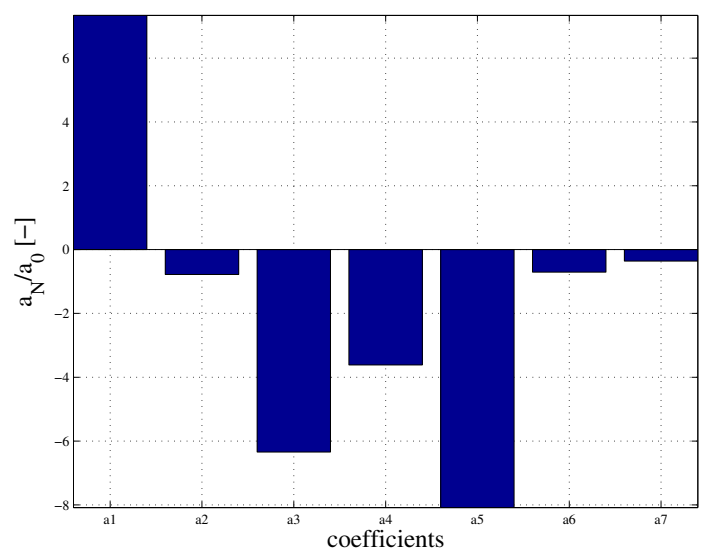

(a) Principle relative effects of the different input parameters.

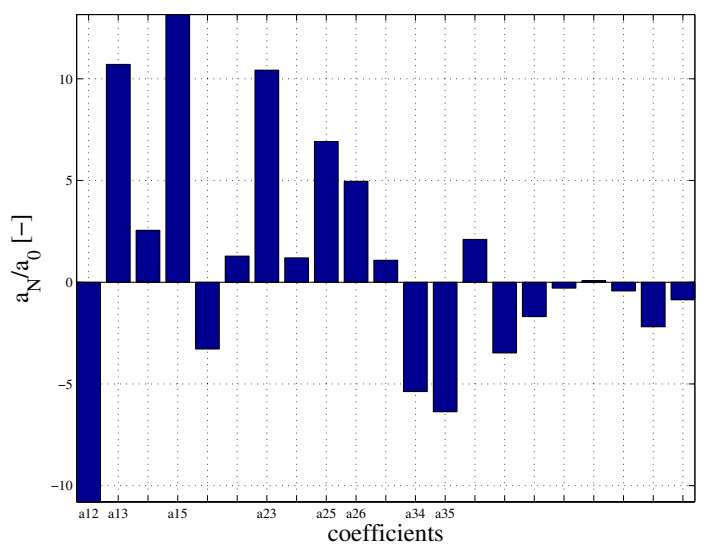

(b) Interaction effects.

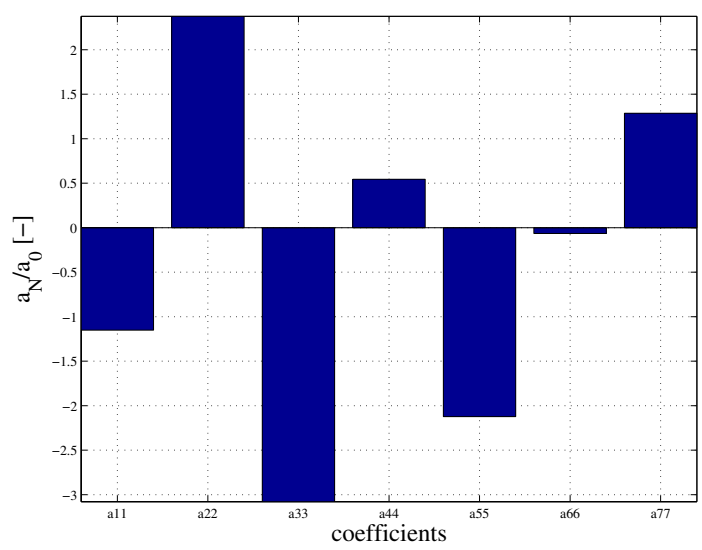

(c) Quadratic effects.

Fig. 3. Linear model coefficients.

Temperature is held constant in experimentation, which is justified as in our application the motor is never in continuous

\begin{tabular}{l|l|l|l|l} 
& Parameter & Symbol & {$[-,+]$} & Unit \\
\hline \hline$a_{1}$ & Phase current & $I_{p h}$ & {$[0.095,0.553]$} & $\mathrm{A}$ \\
$a_{2}$ & Phase voltage & $U_{p h}$ & {$[49.9,122.2]$} & $\mathrm{V}$ \\
$a_{3}$ & Supply current & $I_{t o t}$ & {$[0.6,2.8]$} & $\mathrm{A}$ \\
$a_{4}$ & Torque & $M$ & {$[0,1]$} & $\mathrm{Nm}$ \\
$a_{5}$ & Frequency & $f$ & {$[39.5,44]$} & $\mathrm{kHz}$ \\
$a_{6}$ & Phase shift & $\phi$ & {$\left[\frac{\pi}{90}, \frac{\pi}{2}\right]$} & $\mathrm{rad}$ \\
$a_{7}$ & Supply voltage & $U_{0}$ & {$[15,20]$} & $\mathrm{V}$ \\
\multicolumn{3}{|c}{ TABLE II }
\end{tabular}

PARAMETER DEFINITION FOR THE DESIGNED EXPERIMENT.

motion for longer than a few seconds, and therefore its surface temperature remains stable. This hypothesis is valid only in well defined laboratory conditions and the study presented here must be extended to be of general validity.

As we see from Equation 1, the coefficients of the obtained linear model contain information about the parameter's influence on the output. Referring to Figure 3(a), we see that the parameters with the greatest influence on the speed output are frequency $(f)$, current $\left(I p h\right.$ and $\left.I_{t o t}\right)$ and the applied load torque $(M)$. Figure 3(b) represents the interaction coefficients. Interaction of Iph with $U p h\left(a_{12}\right)$, Itot $\left(a_{13}\right)$ and $f\left(a_{15}\right)$ is important. The interaction of the two current measures being obvious, phase voltage and current are linked as the voltage is a image of the current by the piezoceramic impedance. Amplitude and intensity are then linked to frequency as the piezoceramic impedance is a function of its vibration frequency (cf. Figure 4).
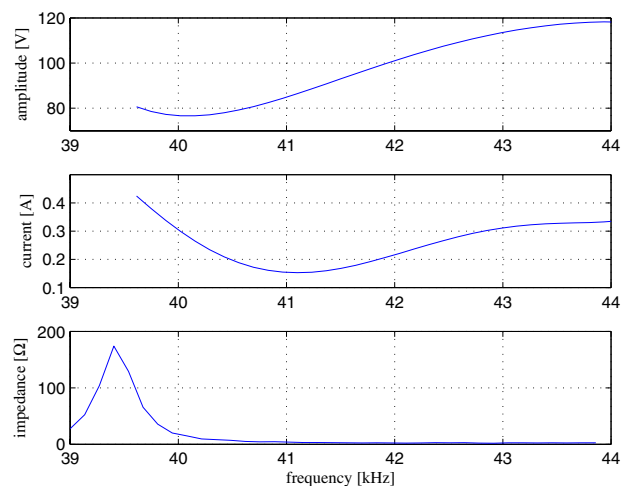

Fig. 4. Impedance as a function of frequency.

From the above observations, it is apparent that frequency and load torque must be taken into account as inputs for the neural network. From the other possible inputs, phase current is selected as a third input parameter. Although its contribution to the output is slightly lower than the supply current's, the correlation with phase and supply voltage is higher.

\section{Response Surface Analysis}

The excitation voltage of the piezoceramics must be chosen to obtain an optimal behavior and is not exploited as main control variable. For speed control we have thus the choice between frequency $f$ and phase shift $\phi$. We are especially 


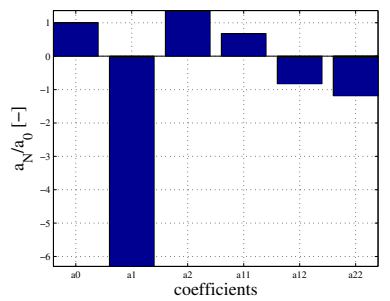

(a) $a_{1} \equiv f$ and $a_{2} \equiv M$.

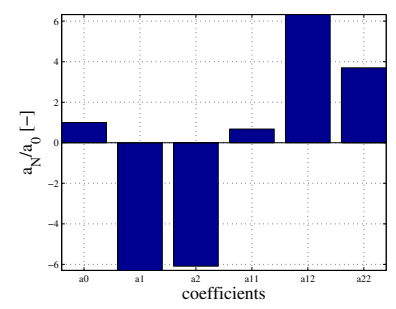

(b) $a_{1} \equiv \phi$ and $a_{2} \equiv M$.
Fig. 5. Coefficients of the response surface models; (a) corresponds to Figure 6 and (b) to Figure 7.

interested in how the motor reacts on an externally applied load torque because this represents the intended working condition. Two studies are therefore necessary :

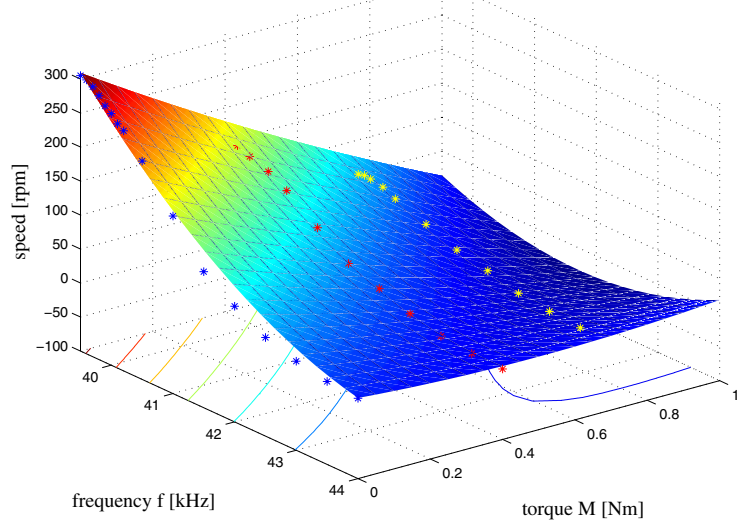

Fig. 6. Response surface : Torque and frequency

- speed output of the motor and the behavior under load when phase shift between the two excitation signals is varied at constant optimal driving frequency

- speed output of the motor and the behavior under load as a function of drive frequency when the phase shift between the two excitation signals is held constant.

In both cases, a set of two parameters $(f, M$ and $\phi, M$ respectively) is chosen to find the model coefficients by the method of least squares (Figure 5). The coefficients are then used to draw the response surface based on the entire normalized input space. The second order model taking into account main effects, interactions and squared terms delivers the best model of the real motor behavior, which was verified with measures outside the central composite design. At three levels of load torque, the respective second model parameter was varied in the region of interest (Figure 7 and Figure 6). The response surface corresponding to phase shift control (Figure 7) does not match reality at high torque for low values of phase shift. Speed becomes negative there, which is not true, but rotor stops due to non-linearity in the rotor-stator surface contact phenomenon. This is known, and dead-zone compensation methods have been proposed [13]. However, for the present application, where high load torque is applied, this

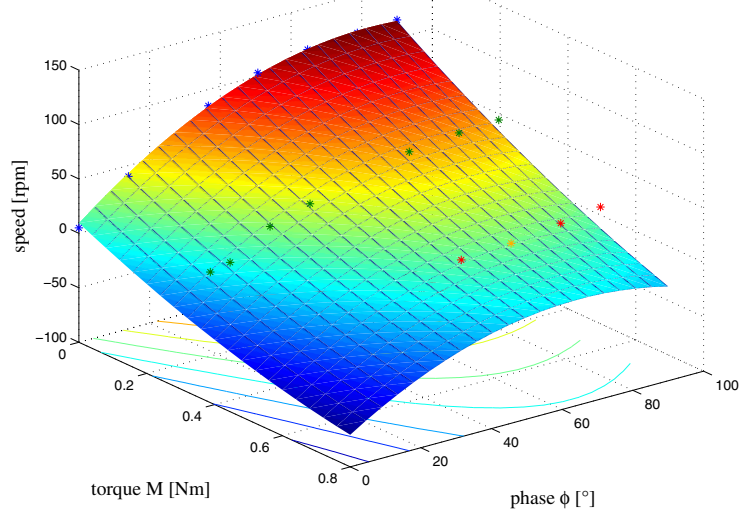

Fig. 7. Response surface : Torque and phase.

dead-zone is not acceptable and we therefore use a different frequency control method. Figure 6 shows that by controlling the frequency on an interval of $40[k H z] \leq f \leq 44[k H z]$, the speed output follows a linear law over the entire working domain (load torque of $\mathrm{M} \geq 0.8 \mathrm{Nm}$ is never applied at normal working conditions).

\section{MOtOR CONTROL}

Now we know that, basically, the speed of the USM can be controlled by varying the frequency, the voltage amplitude and the phase difference of the two sinusoidal input waveforms. However, the USM suffers from severe system nonlinearities and parameter variations due to changes in load torque as well as surface temperature. Therefore, it is not possible to use a basic PID type controller without any precaution. We have developed a controller based on amplitude modulation of the supply voltage. We use the degree of freedom offered by the amplitude to linearize the frequency-speed characteristic chosen as control law in the previous section. The optimal variation of the supply voltage, making it possible to linearize this characteristic, is obtained when the parameter of control $a$ is a function of the frequency according to the diagram shown in Figure 8.

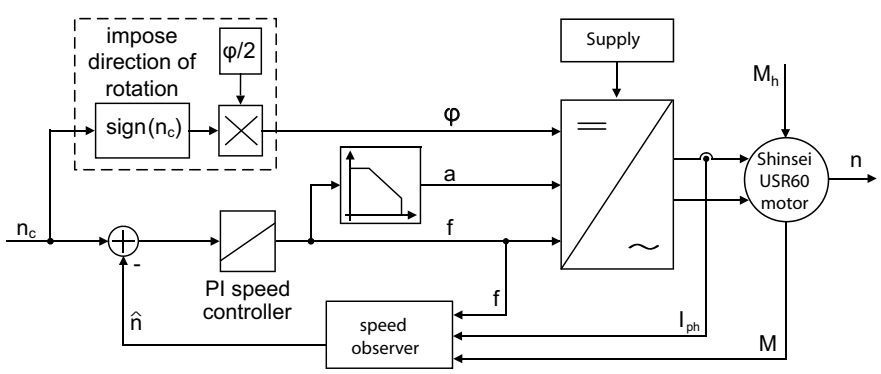

Fig. 8. Schematic representation of the implemented speed controller. 


\section{A. Speed limitation}

As soon as the motor is operated outside the linear domain, it is likely to stop abruptly. Most commonly this happens when the commanded frequency is dropped below antiresonance which varies as a function of applied torque as illustrated in Figure 9. This characteristic is taken into account in the control algorithm in order to prevent this pull-out phenomena.

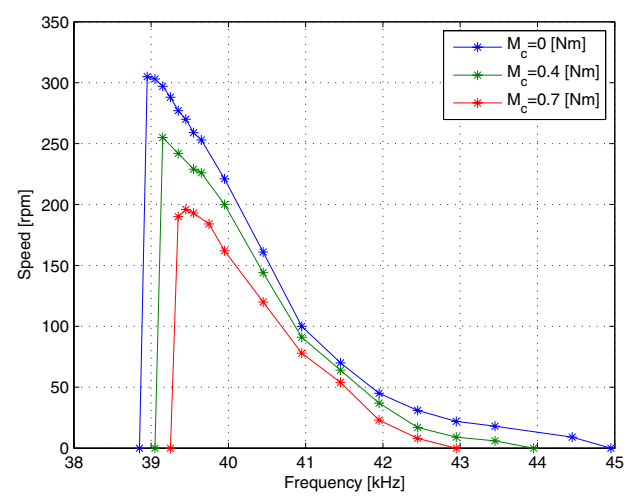

Fig. 9. Change of the frequency-speed characteristic with varying load torque conditions.

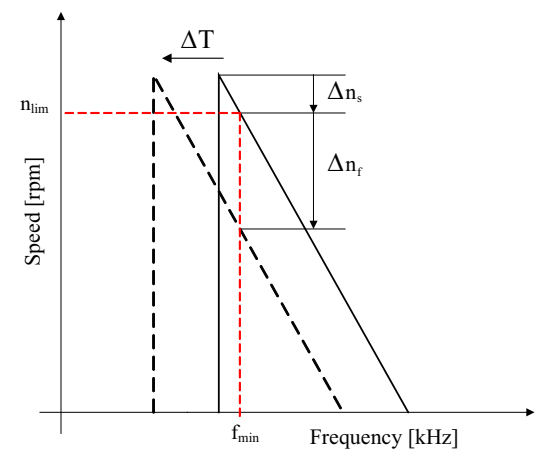

Fig. 10. Speed limitation to prevent pull-off due to changes in the frequencyspeed characteristic.

Figure 10 shows schematically that as we would limit the allowed frequency range by $f_{\min }$, the drift of the resonance frequency induced by tempereture shift $\Delta T$ would decrease the maximum speed by $\Delta n_{f}$. Hence we introduce a speed limit $n_{\text {lim }}$ leaving just a small security gap $\Delta n_{s}$ relating to the resonance peak.

\section{Vi. Speed Estimation Using Artificial NeURal NETWORKS}

Estimating speed instead of using encoders reduces cost and dimensions of the robot and increases reliability. An ANN algorithm is chosen for the purpose of speed estimation because of the nonlinearity of the motor characteristics and the fact that valid analytical models are still too complex to be exploited for control purposes. A neural network, given that enough information is supplied, can be trained to learn any function. Inspired by biologic nervous systems, it is composed of simple elements (neurons) working in parallel which are linked by weighted connections. These weights are adjusted during offline training in order to minimize the error between measured and estimated output.

The study described in the preceding chapters showed that a mathematical relation

$$
n=f_{\hat{n}}\left(f, I_{p h}, M\right)
$$

gives the relationship between motor speed $n$ and frequency $f$, phase current $I_{p h}$ and load torque $M$. Using this function $f_{\hat{n}}$, we are able to estimate motor speed. The ANN is then trained using the DoE data obtained with a test bench as well as experimental data from the prototype aiming to identify this relation. After the training step, the online simulation of the network, excited with measurements of frequency $(f)$, phase current $\left(I_{p h}\right)$ and load torque $(M)$, allows us to estimate speed (Figure 13).

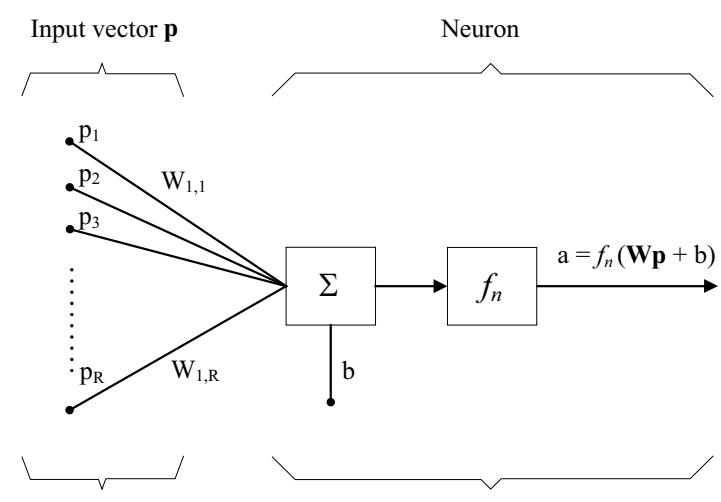

Fig. 11. Neuron model.

The model of the neuron used is represented in Figure 11 , with $R$ the number of elements in the input vector $\mathbf{p}$. Each input is balanced by a corresponding weight $W_{1, R}$. The sum of the balanced inputs, with bias $b$, represents the input of the transfer function $f_{n}$ associated to each neuron. Any differentiable transfer function $f_{n}$ may be used to generate the neuron output.

Several neurons combined in parallel form a layer and multiple layers are called a multi layer ANN. Every element of the input vector $\mathbf{p}$ is connected to every neuron via the matrix W containing the corresponding weights. The neuron model is hence applied for every neuron in the layer to generate the output vector,

$$
\mathbf{a}=f_{n}(\mathbf{W p}+\mathbf{b})
$$

which is the input to the next layer of the network. The structure of the ANN designed to estimate the function given in Equation 7 is shown in Figure 12.

Once the structure is determined, the weights and biases for each neuron must be initialized and training can start. The training process requires a set of input vectors $\mathbf{p}$ and corresponding objective output vector $\mathbf{t}$. During training, the weights and biases are adjusted with the objective of minimizing the performance function 9 . The performance function is defined 


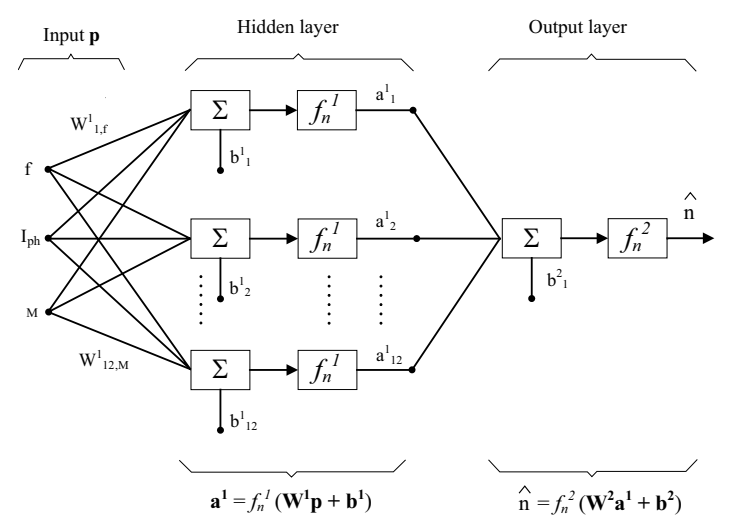

Fig. 12. The ANN is composed of a hidden layer with 12 neurons (sigmoid type transfert function) and a output layer with a single linear neuron.

as the mean of the squared error between network output $\mathbf{a}(k)$ and objective output $\mathbf{t}(k)$ over the length $N$ of one training step. The weights and biases are updated at the end of each training step.

$$
V=\frac{1}{N} \sum_{k=1}^{N}(\mathbf{a}(k)-\mathbf{t}(k))^{2}
$$

The backpropagation algorithm [14] is used to train the ANN. The idea of this algorithm is to calculate the partial derivative of the performance function with respect to each weight of the network. Let $W_{i, j}$ be the weight of any neuronal connection $\mathrm{i}-\mathrm{j}$ of the network, the adjustment $\Delta W_{i, j}$ at the end of every training step is found with

$$
\Delta W_{i, j}=-\alpha \frac{\delta V}{\delta W_{i, j}}
$$

The algorithm calculates then the new value of the connection weight by the following Equation :

$$
W_{i, j}(h+1)=W_{i, j}(h)+\Delta W_{i, j}(h)
$$

where $h$ is the $h^{\text {th }}$ iteration of the backpropagation algorithm.

To find the minimum of the performance function 9, we apply the Levenberg-Marquardt algorithm [15]. The Hessian matrix (second derivatives) is approximated as

$$
\nabla^{2} V=\mathbf{J}^{T} \mathbf{J}
$$

and the gradient can be computed as

$$
\nabla V=\mathbf{J}^{T} \mathbf{e}
$$

where $\mathbf{J}$ is the Jacobian matrix that contains first derivatives of the network errors with respect to the weights and biases, and $\mathbf{e}$ is a vector of network errors. Computing the Jacobian matrix through a standard backpropagation technique is much less complex than computing the Hessian matrix [16].

Finally, with our network structure shown in Figure 12, an analytical approximation of the function $f_{\hat{n}}$ (Equation 7) given by the ANN after being trained, enables speed estimation :

$$
\widehat{n}=f_{n}^{2}\left(\mathbf{W}^{2}\left(f_{n}^{1}\left(\mathbf{W}^{1} \mathbf{p}+\mathbf{b}^{\mathbf{1}}\right)\right)+\mathbf{b}^{2}\right)
$$

\section{Where}

$\mathbf{W}^{\mathbf{1}}$ is the weight matrix for the connection between the hidden layer and the input of the network.

$\mathbf{W}^{2}$ is the weight matrix for the connection between the output layer and the hidden layer of the network.

$\mathbf{b}^{\mathbf{1}}$ is the bias vector of the hidden layer.

$\mathbf{b}^{2}$ is the bias vector of the output layer.

p is the input vector containing here the values of frequency, phase current and load torque as follows :

$$
\mathbf{p}=\left(\begin{array}{c}
f \\
I_{p h} \\
M
\end{array}\right)
$$

The transfer function of the hidden layer (cf. Equation 16) is of sigmoid type, which allows the identification of nonlinearities, while the transfer function of the output layer is of linear type, which means that the output may be outside the interval [-1 1$]$.

$$
\begin{gathered}
f_{n}^{1}(x)=\frac{2}{\left(1+e^{-2 x}\right)}-1 \\
f_{n}^{2}(x)=x
\end{gathered}
$$

\section{EXPERIMENTAL RESULTS}

The ANN is trained offline with the backpropagation method in 500 epochs using the DoE data. To verify the validity of the proposed speed estimation method, the estimated rotor speed is used as actual speed in the PI speed controller (cf. Figure 8). We compare the predicted motor speed $\widehat{n}$ to the measured motor speed for the transient response (Figure 13) and the steady-state response (Figure 14). While the speed prediction error is below 5\% when the temperature remains approximatively constant, the error reaches $10 \%$ when the maximal resonance frequency shift due to motor heating is reached. Hence, the proposed speed estimation method is valid for the application in the haptic feedback device, but is less accurate for continous operation of the USM.

\section{CONCLUSION}

In this paper, we presented a sensorless speed controller for USM using an artificial neural network model for speed estimation. Design of experiments methods make it possible to precisely identify the sensitivity of the speed output on the different input parameters of the USM. This approach is particularly interesting because significant data can be acquired with a minimal number of experiments. Using this data, we designed an ANN modeling the rotor speed of the USM based on frequency, phase current and load torque measures. The implemented speed estimation based on this ANN model was tested for its transient and steady state response. However, while the results were good for the presented application, validity for other USM and different applications has yet to be shown. Therefore, the research will be continued with the aim to generalize the presented speed estimation method. 

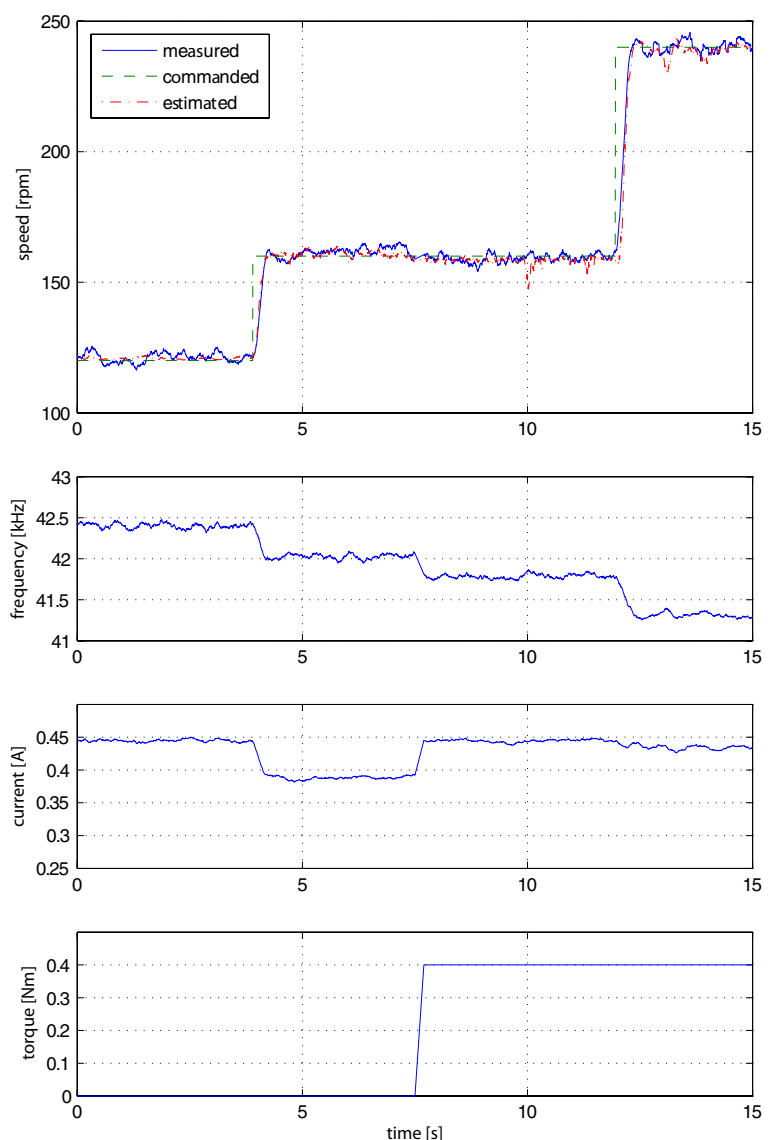

Fig. 13. The results of speed estimation : transient response.

\section{REFERENCES}

[1] M. Flueckiger, M. Bullo, D. Chapuis, R. Gassert, and Y. Perriard, "fmri compatible haptic interface actuated with traveling wave ultrasonic motor," Conference Record of the Fourtieth IAS Annual Meeting, 2005, vol. 3, pp. $2075-2082,2005$.

[2] E. Burdet, R. Gassert, G. Gowrishankar, D. Chapuis, and H. Bleuler, "fMRI compatible haptic interfaces to investigate human motor control," in Springer Tracts in Advanced Robotics, M. Ang and O. Khatib, Eds. Springer Berlin / Heidelberg, 2006, vol. 21, ch. II. Human-Centered Robotics, pp. 25-34.

[3] K. Chinzei, N. Hata, F. Jolesz, and R. Kikinis, "Surgical assist robot for the active navigation in the intraoperative mri : Hardware design issues," Proc. IEEE/RSJ International Conference on Robotics and Intelligent Systems (IROS), pp. 727-732, 2000.

[4] T. Sashida and T. Kenjo, An Introduction to Ultrasonic Motors, O. Science, Ed., 1993.

[5] A. M. Flynn, "Piezoelectric ultrasonic micromotors," Ph.D. dissertation, 1995.

[6] T. Maeno, T. Tsukimoto, and A. Miyake, "Finite-element analysis of the rotor/stator contact in a ring-type ultrasonic motor," IEEE Transactions on Ultrasonics, Ferroelectrics and Frequency Control, vol. 39, no. 6 , pp. 668-674, November 1992.

[7] N. W. Hagood IV and A. J. McFarland, "Modeling of a piezoelectric rotary ultrasonic motor," IEEE transactions on ultrasonics, ferroelectrics and frequency control, vol. 42, no. 2, pp. 210 - 224, 1995.
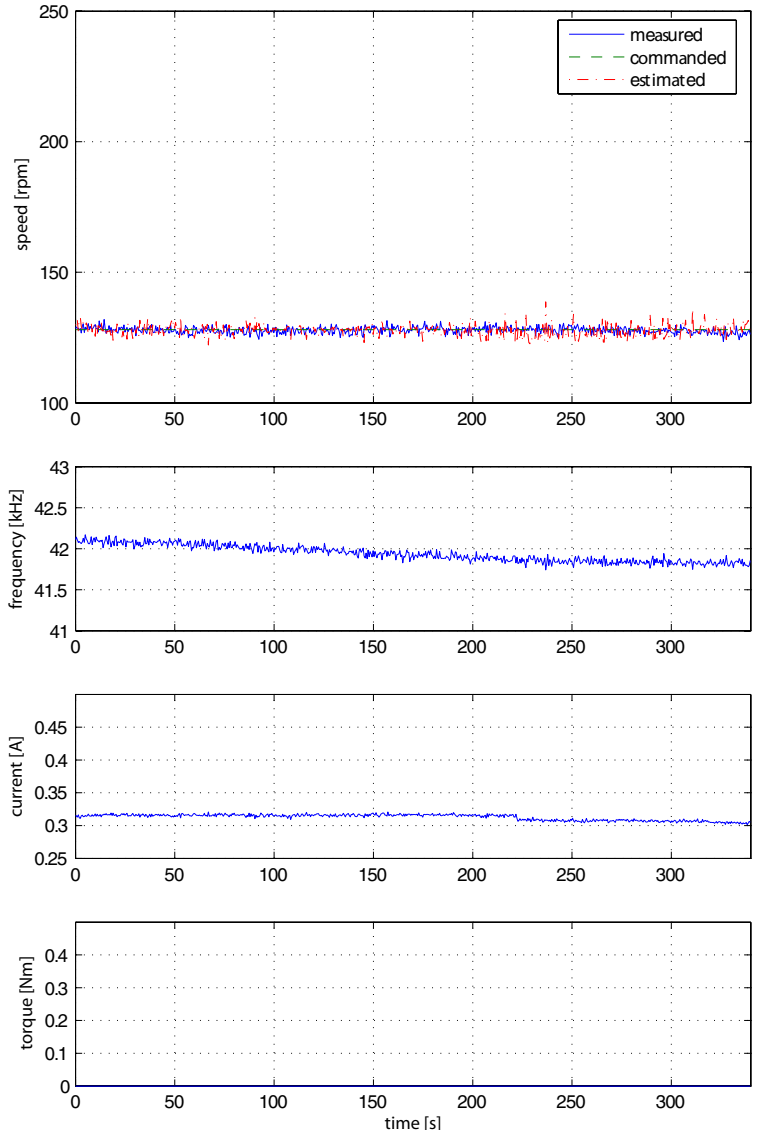

Fig. 14. The results of speed estimation : steady-state response

[8] M. Bullo and Y. Perriard, "Influences to the mechanical performances of the traveling wave ultrasonic motor by varying the prestressing force between stator and rotor," 2003 IEEE Symposium on Ultrasonics, vol. 1, pp. 593 - 596, 2003.

[9] T. Senjyu, T. Yoshida, K. Uezato, N. Urasaki, and S. Panda, "Speed sensorless control of ultrasonic motors using neural networks," 29th Annual Conference of the IEEE Industrial Electronics Society, vol. 1, pp. $335-340,2003$

[10] G. Box, W. Hunter, and J. Hunter, Statistics for Experimenters, An introduction to design, data analysis and model building, J. Wiley, Ed., 1978.

[11] R. G. Miller, Beyond ANOVA : Basics of Applied Statistics., C. . Hall, Ed., 1997.

[12] M. Bullo, "Modélisation et commande du moteur piézoélectrique à onde progressive," Ph.D. dissertation, 2005.

[13] T. Senjyu, T. Kashiwagi, and K. Uezato, "Position control of ultrasonic motors using mrac and dead-zone compensation with fuzzy inference," 32nd Annual IEEE Power Electronics Specialists Conference, vol. 1, pp. 2031 - 2036, 2001.

[14] S. Omatu, M. Khalid, and R. R. Yusof, Neuro-control and its applications, ser. Advances in industrial control. Springer, 1996.

[15] D. M. Bates and D. G. Watts, Nonlinear Regression and Its Applications, Wiley, Ed., New York.

[16] M. T. Hagan and M. B. Menhaj, "Training feedforward networks with the marquardt algorithm," IEEE Transactions on Neural Networks, vol. 5, no. 6, pp. 989 - 993, 1994 